\title{
Cadeia de valor da saúde: um modelo para o sistema de saúde brasileiro
}

\author{
Healthcare value chain: a model for the Brazilian healthcare system
}

Marcelo Caldeira Pedroso ${ }^{1}$

Ana Maria Malik ${ }^{2}$

${ }^{1}$ Departamento de Administração, Faculdade de Economia, Administração e Contabilidade,

Universidade de São Paulo. Av. Prof. Luciano Gualberto 908, Cidade Universitária. 05508-010 São Paulo SP. mpedroso@usp.br

${ }^{2}$ Departamento de Gestão Pública, Escola de

Administração de Empresas de São Paulo, Fundação

Getúlio Vargas.
Abstract This article presents a model of the healthcare value chain which consists of a schematic representation of the Brazilian healthcare system. The proposed model is adapted for the Brazilian reality and has the scope and flexibility for use in academic activities and analysis of the healthcare sector in Brazil. It places emphasis on three components: the main activities of the value chain, grouped in vertical and horizontal links; the mission of each link and the main value chain flows. The proposed model consists of six vertical and three horizontal links, amounting to nine. These are: knowledge development; supply of products and technologies; healthcare services; financial intermediation; healthcare financing; healthcare consumption; regulation; distribution of healthcare products; and complementary and support services. Four flows can be used to analyze the value chain: knowledge and innovation; products and services; financial; and information.

Key words Healthcare management, Strategic management, Healthcare services, Value chain
Resumo Este artigo apresenta um modelo de cadeia de valor da saúde que representa, de maneira esquemática, o sistema de saúde do Brasil. O modelo proposto tem como intuito apresentar uma adequação à realidade brasileira, bem como abrangência e flexibilidade para utilização em atividades acadêmicas e análises do setor de saúde do Brasil. O modelo coloca ênfase em três componentes: principais atividades dessa cadeia, agrupadas em elos verticais e horizontais; missão de cada um desses elos; e principais fluxos da cadeia. A cadeia proposta é formada por seis elos verticais $e$ três horizontais, perfazendo um total de nove: desenvolvimento de conhecimento em saúde; fornecimento de produtos e tecnologias; serviços de saúde; intermediação financeira; financiamento da saúde; consumo de saúde; regulação; distribuição de produtos de saúde; e serviços de apoio e complementares. A análise da cadeia proposta pode ser realizada por meio de quatro fluxos: inovação e conhecimento; produtos e serviços; financeiro; $e$ de informação.

Palavras-chave Gestão em saúde, Gestão estratégica, Serviços de saúde, Cadeia de valor 


\section{Introdução}

Este artigo apresenta um modelo de cadeia de valor da saúde com o intuito de representar, de maneira esquemática, o sistema de saúde do Brasil. A primeira etapa para caracterizar este modelo consiste em delimitar três conceitos: valor, cadeia de valor e escopo da saúde.

Na saúde, valor é um conceito com diferentes conotações, o que dificulta sua compreensão e adequada utilização. Além disso, o valor na saúde é relativamente pouco mensurado ${ }^{1}$. O valor pode ser definido como a diferença entre os benefícios auferidos pelos clientes que compram os produtos e serviços e os custos econômicos para a empresa que os fabrica e entrega. Assim, o valor equivale ao conceito econômico de excedente total, que é igual à soma dos excedentes do produtor e do consumidor ${ }^{2}$.

$\mathrm{Na}$ saúde, pode-se dizer que essa definição apresenta uma aplicação limitada, por ser focada na dimensão econômica. Nesse setor, o "valor social" está inexoravelmente imbricado aos seus objetivos. Esse valor é criado quando os recursos, os processos, os produtos e os serviços de uma organização são utilizados para gerar melhoria nas vidas dos indivíduos ou da sociedade como um todo, adicionalmente ao valor econômico. O "valor social", na forma de melhoria da saúde da população, é um dos objetivos fundamentais de um sistema de saúde ${ }^{3}$. No Brasil, segundo sua Constituição, a saúde é considerada um direito social ${ }^{4}$.

Além disso, os objetivos sociais estão entre os elementos que justificam a existência de organizações sem fins lucrativos (ex.: hospitais filantrópicos). Organizações com fins lucrativos também deveriam considerar o "valor social" como parte integrante de seus objetivos. Essa questão está associada ao conceito de responsabilidade social, em que as empresas devem se preocupar concomitantemente com a obtenção de resultados econômicos positivos e a geração de bem estar social. Alguns autores sugerem a denominação "valor compartilhado", que denota a capacidade de uma organização aumentar sua competitividade e, simultaneamente, proporcionar melhorias nas condições econômicas e sociais da comunidade em que ela convive ${ }^{5}$. Nesse sentido, este trabalho adota uma definição expandida de valor, por meio da consideração simultânea dos benefícios econômicos e sociais.

A cadeia de valor interna (ou intraempresa) consiste no conjunto de atividades primárias e de suporte realizadas para projetar, produzir e entregar um produto ou serviço ${ }^{6}$. A cadeia de valor externa (ou estendida) contempla o conjunto de atividades que gerencia e executa os fluxos de produtos, os serviços e os recursos (conhecimento, financeiro e informação) entre as empresas participantes dessa cadeia, até atingir os consumidores finais ${ }^{7}$. Os fluxos principais em cadeia de valor (produtos e serviços, conhecimento, financeiro e informação) foram adaptados ao modelo de cadeia de valor da saúde proposto neste artigo.

A literatura apresenta várias definições de saúde, que abordam desde um sentido estrito (saúde como ausência de doença) ${ }^{8}$ até uma visão integrada (saúde como estado de bem-estar físico, mental e social) ${ }^{9}$. Pode-se interpretar a saúde como um continuum que passa por diferentes modalidades de normalidade no estado de bemestar (saúde positiva) e diferentes severidades de doença (saúde subótima) ${ }^{8}$.

O presente trabalho considera a saúde desde a perspectiva positiva (que engloba os elementos de bem estar e promoção da saúde) até a patológica (que aborda a doença e seus fatores de risco). Ainda considera seu escopo segundo uma concepção integral: o conjunto de atividades e recursos alocados à promoção e proteção da saúde, prevenção de doenças, diagnóstico, tratamento, reabilitação, monitoramento e gestão de doenças crônicas.

A seguir, é apresentada uma revisão de literatura sobre cadeia de valor no setor de saúde. Após esta revisão, faz-se uma proposição de representação esquemática dessa cadeia. Na sequência, são discutidos seus quatro fluxos: inovação e conhecimento, produtos e serviços, financeiro e de informação. Ao final, o trabalho apresenta uma discussão sobre a utilização do modelo proposto em estudos do setor de saúde do Brasil.

\section{Revisão da literatura: cadeia de valor da saúde}

A literatura recente em gestão em saúde apresenta algumas abordagens para a caracterização da cadeia de valor da saúde.

Burns et al. ${ }^{10}$ apresentam um modelo de cadeia de valor para o sistema de saúde norte-americano. Este modelo é formado por cinco elementos principais: (1) pagadores do sistema de saúde, que incluem governo, empresas, indivíduos e agrupamentos de empresas; (2) intermediários financeiros, que contemplam empresas de seguro saúde, organizações de manutenção da saúde 
(HMOs) e de administração de benefícios farmacêuticos; (3) provedores de serviços e produtos de saúde, que abrangem hospitais, médicos, redes integradas de serviços de saúde e farmácias; (4) compradores, que incluem distribuidores de produtos de saúde e organizações de compra; (5) fabricantes, que contemplam indústria farmacêutica, fabricantes de equipamentos de saúde e fabricantes de produtos médicos e cirúrgicos.

Em trabalho posterior, Burns ${ }^{11}$ incorpora os provedores de tecnologia da informação no elo dos fabricantes. Isso se justifica pelo tamanho do mercado de tecnologia da informação na saúde, sua relevância para as organizações de saúde e pacientes, bem como pelo fato desses provedores serem uma das fontes de inovação na cadeia de valor. Esse autor considera dois fluxos críticos nessa cadeia: o financeiro e o de inovação. Quanto ao primeiro, ele aponta que se propaga do lado dos pagadores (governo, empresas e indivíduos) para o lado dos fabricantes. Em relação ao segundo, o sentido é inverso. Assim, a inovação flui dos fabricantes para os elos adjacentes da cadeia de valor. O elo dos provedores de serviços e produtos de saúde define grande parte dos gastos e do consumo em saúde. Dessa forma, segundo Burns ${ }^{11}$, os prestadores de serviços (principalmente médicos e hospitais) determinam quanto da inovação é necessário e possível utilizar no tratamento dos pacientes e, ao mesmo tempo, consideram as limitações de recursos financeiros provenientes dos pagadores.

Porter e Teisberg ${ }^{12}$ discutem o sistema de saúde norte-americano e apresentam proposições com base no referencial teórico de cadeia de valor e estratégia competitiva. A cadeia utilizada por esses autores foca nas principais atividades de assistência à saúde. Eles adotam seis atividades primárias (monitorar/prevenir, diagnosticar, preparar, intervir, recuperar/reabilitar e monitorar/gerenciar) e quatro de suporte (desenvolver conhecimento, informar, mensurar e prover acesso).

Para eles, o problema atual do sistema de saúde norte-americano não está na falta de competição, mas sim no tipo errado de competição. Segundo esses autores, o sistema de saúde dos EUA gravitou para uma competição de soma zero, na qual os ganhos de um participante do sistema ocorrem em detrimento dos demais; os participantes competem, por isso, com base na transferência de custos e ineficiências de uns para os outros. Essa forma de competição é causada principalmente pela falta de alinhamento de objetivos e incentivos na cadeia de valor. Eles recomendam um realinhamento na cadeia, de modo que a competição seja direcionada para oferecer valor aos pacientes. Para eles, valor na saúde significa o resultado obtido na saúde do cidadão por recurso investido. Assim, se todos os participantes competirem com base no valor, este deverá aumentar para todos.

Christensen et al. ${ }^{13}$ discutem o sistema de saúde norte-americano e apresentam proposições com base no referencial teórico da inovação de ruptura. Uma das proposições consiste em uma nova configuração da cadeia de valor da saúde, cuja premissa principal funda-se no alinhamento dos seus diversos participantes. Os autores apresentam uma cadeia com três fluxos: de dados, financeiro e contratual. Os participantes são indivíduos, hospitais gerais, médicos autônomos, diferentes modelos de clínicas (especializadas, gerais e de pronto-atendimento), redes facilitadoras, empregadores e redes integradas de saúde (equivalentes, no Brasil, às modalidades de medicinas de grupo e cooperativas). A proposição de cadeia de valor desses autores contempla os seguintes elementos: (1) os indivíduos são posicionados no centro da cadeia e os fluxos de dados dos demais participantes fluem para o prontuário eletrônico do paciente; (2) os indivíduos possuem planos com coparticipação e franquia, além de poupança saúde (HSA - health savings account); (3) os empregadores realizam contratos diretamente com os provedores de serviços de saúde; (4) as redes integradas de saúde têm responsabilidade financeira pelo tratamento dos doentes crônicos; (5) há diferentes modelos de pagamentos: as redes integradas recebem por afiliação; os médicos autônomos e as clínicas especializadas recebem por serviço; as clínicas gerais e de pronto-atendimento recebem por resultado; e os hospitais gerais recebem segundo diferentes modelos de remuneração.

Pitta e Laric ${ }^{14}$ apresentam um modelo de cadeia de valor da saúde baseado nos principais participantes deste setor. Segundo esses autores, a cadeia de valor não é linear ou sequencial; os relacionamentos entre os stakeholders podem ser circulares ou iterativos. Os autores consideram oito grupos de participantes: (1) pacientes; (2) médicos; (3) farmácias, fornecedores de equipamentos médicos, prestadores de serviços médicos e de medicina alternativa; (4) hospitais; (5) operadoras de planos de saúde; (6) empregadores; (7) governo; e (8) fabricantes de produtos farmacêuticos.

Pisano $^{15}$ utiliza um modelo de cadeia de valor, denominado anatomia do negócio de biotecnologia, para analisar o desempenho do se- 
tor. Segundo esse autor, o termo anatomia contempla três elementos principais: (1) participantes do setor (ex.: empresas novas e estabelecidas, universidades, investidores, clientes); (2) esquemas institucionais que conectam esses atores (ex.: mercados de capital, propriedade intelectual e produtos) e (3) normas que regem o funcionamento desses esquemas institucionais (ex.: regulamentação, governança corporativa, direitos de propriedade intelectual). Ele foca sua análise na transformação da ciência e do conhecimento em produtos biotecnológicos. Nesse sentido, o conhecimento em biotecnologia é o principal elemento de entrada. Esse conhecimento é transformado em produtos entregues aos consumidores e utilizados por eles.

Outros autores abordam a cadeia de valor da saúde no Brasil. Gadelha ${ }^{16}$ apresenta um modelo conceitual sobre o denominado complexo industrial da saúde. Ele considera três grupos como seus principais componentes: (1) indústrias de base química e biotecnológica, compostas pela indústria de fármacos e medicamentos, vacinas, hemoderivados e reagentes para diagnóstico; (2) indústrias de base mecânica, eletrônica e de materiais, que abordam a indústria de equipamentos mecânicos, eletroeletrônicos, próteses e órteses, e materiais de consumo; (3) prestadores de serviços, que contemplam os hospitais, os ambulatórios e os serviços de diagnóstico e tratamento. Segundo o autor, o complexo industrial está inserido em um contexto político e institucional cujos principais atores são Estado, instituições de Ciência e Tecnologia, sociedade e população.

Campos et al. ${ }^{17}$ utilizam a cadeia de valor como referencial teórico para apresentar uma proposta de reorganização da atenção na saúde suplementar no Brasil. Esses autores consideram dez conjuntos de participantes da cadeia de valor da saúde no Brasil: (1) operadoras de planos de saúde; (2) médicos e outros profissionais de saúde; (3) hospitais; (4) serviços de apoio ao diagnóstico e terapia; (5) indústria farmacêutica; (6) distribuidores de medicamentos; (7) farmácias; (8) indústria de equipamentos e materiais; (9) distribuidores de equipamentos e materiais; e (10) serviços do governo. Segundo esses autores, além destes dez participantes, há o cliente final (governo, famílias e empresas) que está posicionado no centro da cadeia de valor da saúde. Os autores propõem uma reorganização nesta por meio de um pacto entre seus participantes. Essa proposta é baseada em quatro principais elementos: (1) modelo de atenção à saúde baseado em protocolos ou acordos de desempenho, e coordenado pelas operadoras de planos de saúde; (2) transparência de resultados, por meio da publicação sistemática de resultados da assistência à saúde; (3) pagamento por desempenho aos prestadores de serviços de saúde; (4) regulação, com ênfase na manutenção da integralidade da atenção à saúde.

Outros trabalhos abordam o tema, sem apresentar uma proposta de cadeia de valor peculiar para a saúde. Por exemplo, Walters e Jones ${ }^{18}$ utilizam os conceitos de cadeia de valor em um estudo de caso no Hospital Queen Elizabeth (Nova Zelândia). No entanto, os autores consideram que a cadeia de valor da saúde é, em sua essência, similar a cadeias de outros setores - do que discordamos. Consideramos que o setor de saúde apresenta particularidades que demandam uma abordagem única: assim, há a necessidade de se considerar uma cadeia de valor específica. Nessa questão, Pedroso ${ }^{19}$ apresenta sete características que, em seu conjunto, denotam a especificidade do setor.

Herzlinger $^{20,21}$ não apresenta um modelo explícito de cadeia de valor, mas utiliza seus conceitos para analisar o sistema de saúde norte-americano. Em seus trabalhos, ela sugere que a cadeia de valor da saúde seja direcionada pelos consumidores. Essa recomendação é baseada no conceito de consumismo em saúde segundo o qual os recursos sociais (incluída a assistência à saúde) deveriam ser alocados preferencialmente segundo decisões individuais e não coletivas.

\section{Por que apresentar outro modelo de cadeia de valor da saúde?}

Os autores consideram duas justificativas principais para propor um novo modelo de cadeia de valor da saúde: (1) adequação à realidade brasileira; (2) abrangência e flexibilidade para utilização em atividades acadêmicas e análises do setor de saúde do Brasil.

Parte dos modelos foi desenvolvida tendo como referência o sistema de saúde dos Estados Unidos. No entanto, alguns pesquisadores consideram difícil estabelecer um paralelo entre o sistema de saúde dos EUA e os de outros países, em função da singularidade do norte americano ${ }^{22}$.

Deve-se considerar que há algumas similaridades entre o sistema de saúde brasileiro e o norte americano, tais como: (1) estrutura de financiamento: no Brasil, $42 \%$ do financiamento é público e 58\% privado, distribuição é similar à dos Estados Unidos (45\% público e 55\% privado) ${ }^{23}$; (2) estrutura da oferta de serviços: tanto nos Estados Unidos ${ }^{22,24}$ quanto no Brasil, a maior parte 
da oferta de serviços de saúde é de propriedade privada (no Brasil, 62\% dos estabelecimentos com internação, 68\% dos leitos hospitalares e 92\% das unidades prestadoras de serviços de medicina diagnóstica são privados ${ }^{25}$ ).

Por outro lado, o contexto do sistema de saúde brasileiro é diferente do norte americano, particularmente nos seguintes elementos: (1) universalidade: no Brasil, o sistema público de saúde (SUS) é universal, o que não ocorre até o $1^{\circ}$. trimestre de 2011 nos Estados Unidos (uma questão que é alvo de constantes debates ${ }^{26}$ ); (2) estrutura econômica: os preços dos produtos e serviços nos Estados Unidos são destacadamente elevados quando comparados com outros países (sendo, portanto, um fator relevante nos gastos em saúde ${ }^{27}$ ).

Assim, muito embora ocorram similaridades entre ambos os sistemas de saúde, há importantes diferenças entre eles. Os modelos de cadeia de valor da saúde apresentados por Burns et al. ${ }^{10}$, Porter e Teisberg ${ }^{12}$, Christensen et al. ${ }^{13}$, Pitta e Laric $^{14}$ e Pisano ${ }^{15}$ foram desenvolvidos tendo como referência o dos Estados Unidos. Dessa forma, eles não estão completamente adaptados à realidade brasileira.

Os trabalhos de Gadelha ${ }^{16}$ e Campos et al. ${ }^{17}$ abordam o sistema de saúde do Brasil. Ambos apresentam contribuição ao tema: Gadelha ${ }^{16}$ por meio de um relevante modelo do complexo industrial da saúde; e Campos et al. ${ }^{17}$, com uma abordagem abrangente e de natureza prática. $\mathrm{O}$ modelo proposto neste artigo (apresentado no tópico seguinte) difere das colocações desses autores em função de duas questões principais: (1) consideração de atividades na cadeia de valor (e não de seus participantes); (2) maior abrangência.

A cadeia de valor da saúde de Porter e Teisberg $^{12}$ é representada por meio de atividades - o que não ocorre com os demais modelos pesquisados, que adotam uma classificação segundo empresas participantes. A caracterização por meio de atividades permite maior generalização e flexibilidade de aplicação, em particular na identificação do escopo de atuação de uma determina organização (que pode ser realizada independentemente de sua classificação na cadeia de valor). Por exemplo, empresas de medicina de grupo e cooperativas podem atuar tanto na intermediação financeira quanto na prestação de serviços. Alguns hospitais filantrópicos de referência atuam tanto na assistência à saúde, quanto em ensino e pesquisa. $\mathrm{Na}$ cadeia proposta no presente trabalho, fica explícita a atuação dessas organizações em elos distintos.
Os modelos de Porter e Teisberg ${ }^{12}$, Pisano ${ }^{15}$, Gadelha ${ }^{16}$ e Campos et al. ${ }^{17}$ apresentam uma abrangência mais restrita, quando comparados com os demais modelos. Porter e Teisberg ${ }^{12}$ focam nas atividades de assistência à saúde; Pisano ${ }^{15}$ analisa o setor de biotecnologia; Gadelha ${ }^{16}$ enfatiza o complexo industrial da saúde; e Campos et al. ${ }^{17}$ abordam a saúde suplementar. O modelo proposto adota uma abordagem abrangente, uma vez que utiliza como referência outros modelos também abrangentes (Burns et al. ${ }^{10}$, Christensen et al. ${ }^{13}$ e Pitta e Laric $\left.{ }^{14}\right)$.

\section{Metodologia adotada}

A cadeia de valor da saúde proposta neste trabalho é resultante de uma abordagem dedutiva, baseada na revisão da literatura sobre esse tema. Foram utilizadas as palavras-chave "value chain" e "healthcare" (ou "health care") para pesquisa nas bases de dados da BVS/Bireme e PubMed/ Medline. Os trabalhos encontrados foram préselecionados com base no título. A seguir, os trabalhos foram filtrados por meio da leitura dos resumos. A leitura do corpo destes trabalhos resultou na seleção final de alguns dos textos apresentados. Adicionalmente os autores incorporaram na proposta seus conhecimentos e experiências prévias em sistema de saúde, bem como na cadeia de valor aplicada a outros setores.

O modelo original foi desenvolvido por um dos autores em 2007. Desde então, ele vem sendo apresentado e discutido sucessivamente com outros pesquisadores e alunos de pós-graduação, em várias aulas e palestras sobre gestão no setor de saúde. Além disso, o modelo foi utilizado em tese de doutoramento na área, sendo também submetido a um processo formal de avaliação. Advindas dessas interações, diferentes contribuições foram incorporadas no modelo original, que resultou na proposta apresentada a seguir.

\section{Cadeia de valor da saúde: uma proposição}

A cadeia de valor da saúde proposta nesse trabalho (parte central da Figura 1) é formada por seis elos verticais e três horizontais, perfazendo um total de nove. Cada um desses agrega um conjunto de atividades com missões específicas. O fluxo das atividades percorre esses elos até a entrega de produtos e serviços aos consumidores, o elo final da cadeia de valor. Dessa forma, o processo de agregação de valor aos pacientes tem início no elo localizado na porção inicial (tam- 


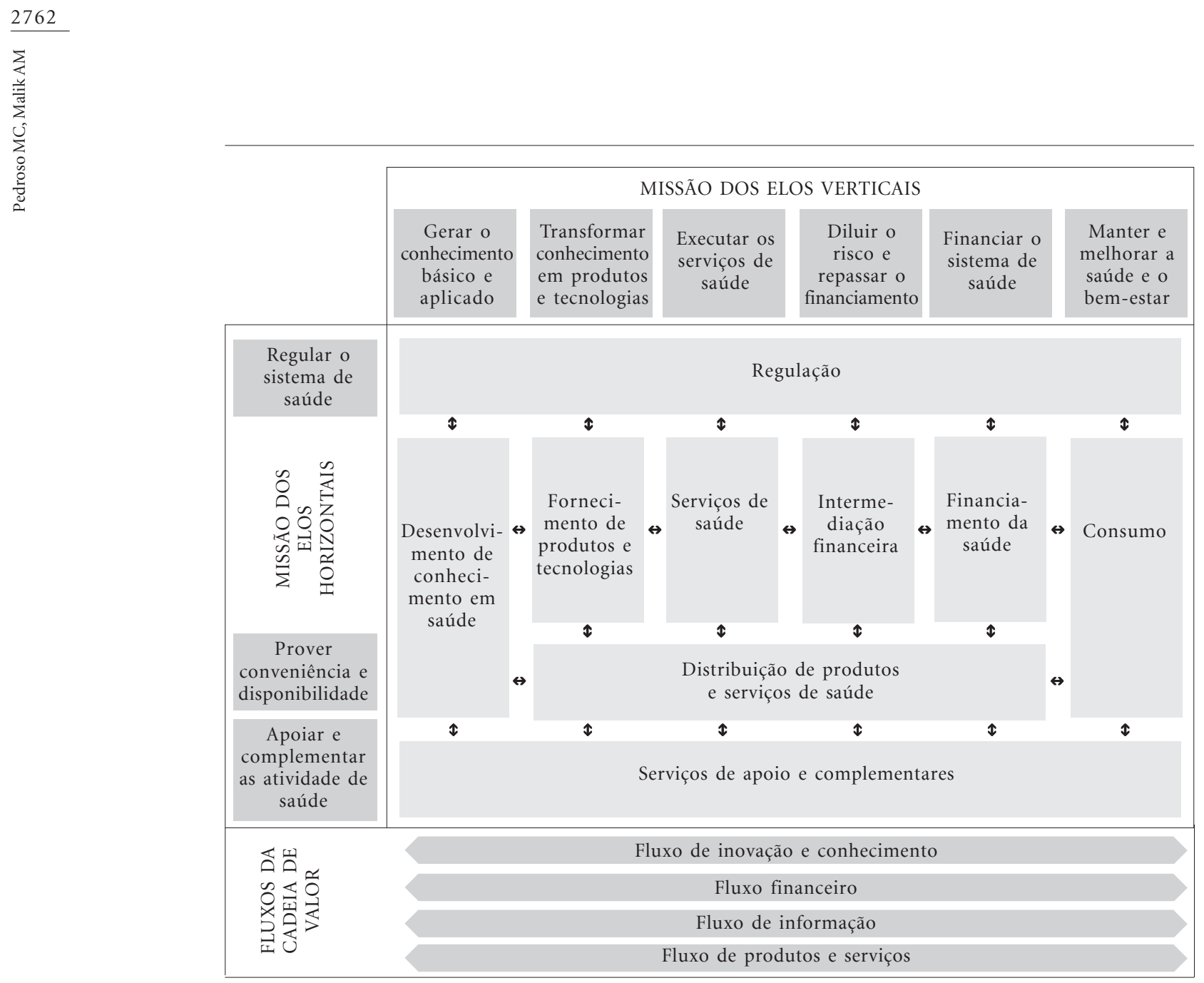

Figura 1. Cadeia de valor da saúde (Pedroso $\left.{ }^{19}\right)$.

bém denominada à montante ou upstream) e se move para a porção final (também denominada à jusante ou downstream). Elos horizontais contemplam atividades que permeiam os verticais e seus objetivos são: regular as atividades do setor de saúde, distribuir os produtos de saúde e executar atividades que apóiam ou complementam as missões de cada um dos elos verticais.

O conhecimento em saúde é o principal insumo na porção inicial da cadeia e é gerado no primeiro elo vertical, o do desenvolvimento de conhecimento em saúde. Este é representado pelas atividades de ensino e pesquisa, básica e aplicada. A missão desse elo é gerar e disseminar esse conhecimento, que é transformado e incorporado em produtos (ex.: medicamentos, materiais médicos) e tecnologias (ex.: equipamentos médico-hospitalares, tecnologia da informação) no elo seguinte.

O segundo elo vertical contempla o fornecimento de produtos e tecnologias de saúde, tais como produtos farmacêuticos sintéticos e bio- tecnológicos, equipamentos médico-hospitalares, órteses, próteses e materiais médicos, além da tecnologia da informação. Sua missão principal consiste na incorporação do conhecimento em produtos e tecnologias, entregues ao próximo elo.

O terceiro elo vertical consiste nos serviços de saúde. Ele aloca os recursos financeiros colocados à disposição pelo elo seguinte (o da intermediação financeira), utiliza a infraestrutura disponível nele próprio (ex.: instalações hospitalares) e consome os recursos oferecidos pelos anteriores (ex.: conhecimento, medicamentos e materiais médicos). Assim, sua missão é utilizar os recursos disponíveis para a prestação de serviços aos consumidores finais. Esse elo é composto pelas atividades de promoção de saúde, proteção da saúde e prevenção de doenças, diagnóstico, tratamento, reabilitação, monitoramento e gestão de doenças crônicas.

O quarto elo vertical considera a intermediação financeira no sistema de saúde, sendo repre- 
sentado pela intermediação dos subsistemas público (SUS) e privado (Saúde Suplementar), além dos gastos diretos das empresas e indivíduos (nesse caso, sem intermediação). Sua missão é repassar a captação financeira aos elos anteriores da cadeia, além de diluir os riscos. O subsistema privado incorpora as atividades de intermediação realizadas pelas operadoras de saúde em suas diferentes modalidades (ex.: seguro saúde, autogestão, medicina de grupo, cooperativas médicas e filantrópicas). Neste subsistema, esse elo representa o fluxo monetário das operadoras de saúde aos elos verticais anteriores e horizontais. Em operadoras de medicina de grupo e cooperativas médicas, quando atuam como redes verticalizadas, o fluxo monetário considera o consumo de recursos da própria organização (ex.: hospitais próprios) ou os pagamentos à rede credenciada. No subsistema público, esse elo representa os pagamentos do SUS às organizações públicas e privadas que participam dos elos verticais anteriores (ex.: provedores de serviços e fornecedores de produtos e tecnologias) e horizontais.

O quinto elo vertical corresponde ao financiamento da saúde, equivalente aos gastos totais de saúde. $\mathrm{O}$ financiamento pode ser público ou privado. Este último, por sua vez, é desdobrado nos gastos dos empregadores, das famílias e dos indivíduos. Nesse sentido, esses três atores - governo, empregadores e indivíduos - são os responsáveis pelos gastos totais de saúde. Sua missão é alocar os recursos financeiros ao sistema de saúde.

O sexto elo vertical (o final da cadeia de valor) consiste no consumo de produtos e serviços de saúde que é realizado pelos pacientes, pessoas saudáveis e empresas. O objetivo dos consumidores é manter e melhorar sua própria condição de saúde e bem-estar (ou de seus funcionários, no caso dos empregadores). Para maximizar o valor entregue aos consumidores, dever-se-ia buscar o alinhamento dos incentivos de todos os participantes da cadeia de valor da saúde.

Adicionalmente, três elos horizontais permeiam os verticais acima descritos. O primeiro deles contempla a regulação da saúde, que se refere às atividades de definição de normas, controle e fiscalização dos produtos e serviços que podem impactar a saúde da população. Tais atividades são realizadas pelas agências reguladoras nacionais (Anvisa e ANS) ${ }^{28}$ e internacionais (FDA nos EUA, e EMEA na Comunidade Européia). Nesse sentido, a missão desse elo consiste em regular as diferentes atividades relacionadas aos produtos e serviços de saúde. No Brasil, por exemplo, a Anvisa foca o controle sanitário da produção e comercialização de produtos e serviços de saúde, enquanto a ANS exerce atividades reguladoras sobre os provedores de planos privados de saúde.

O segundo elo horizontal aborda as atividades de distribuição de produtos e serviços de saúde. Ele é composto pela distribuição de produtos médicos e farmacêuticos, comercialização de serviços (ex.: venda de planos de saúde) e atividades inerentes ao varejo especializado (ex.: drogarias e varejo de produtos médicos hospitalares) e generalista. Sua missão é comercializar os serviços e distribuir os produtos utilizados no setor e, dessa forma, agregar valor de conveniência e disponibilidade.

O terceiro elo horizontal considera os serviços de apoio e complementares, tais como serviços gerais, financeiros, tributários, jurídicos, logísticos, de tecnologia da informação, manutenção, engenharia clínica e pesquisa clínica. Cabelhe apoiar ou complementar as atividades dos demais.

A Figura 2 apresenta uma visão geral dos elos da cadeia de valor da saúde, incluindo as principais atividades de cada um deles.

Os elos da cadeia de valor representam atividades. Nesse sentido, as organizações do setor de saúde decidem atuar em um ou mais deles, bem como realizar uma ou mais atividades. Assim, uma empresa especializada tende a focar em um elo, e por vezes em algumas atividades específicas dentro dele. Por exemplo, uma empresa de medicina diagnóstica pode ser focada somente nessa atividade (e, por vezes, dedicada especificamente a análises clínicas ou ao diagnóstico por imagem). Outras organizações podem atuar somente em um elo, porém com oferta ampliada de produtos ou serviços. Esse é o caso de empresas participantes do elo de fornecimento de produtos e tecnologias que oferecem equipamentos médico-hospitalares, produtos farmacêuticos sintéticos, e biotecnológicos.

Outras empresas podem atuar em mais de um elo simultaneamente. Exemplos incluem as operadoras de saúde nas modalidades medicina de grupo e cooperativa médica (quando atuam na intermediação financeira e na execução de serviços de saúde) e alguns hospitais filantrópicos de referência (que realizam atividades de ensino e pesquisa). Por fim, um paciente também pode assumir papéis em mais de um elo. No caso do pagamento direto por produtos e serviços de saúde, ele atua nas atividades de consumo, financiamento e intermediação financeira. 


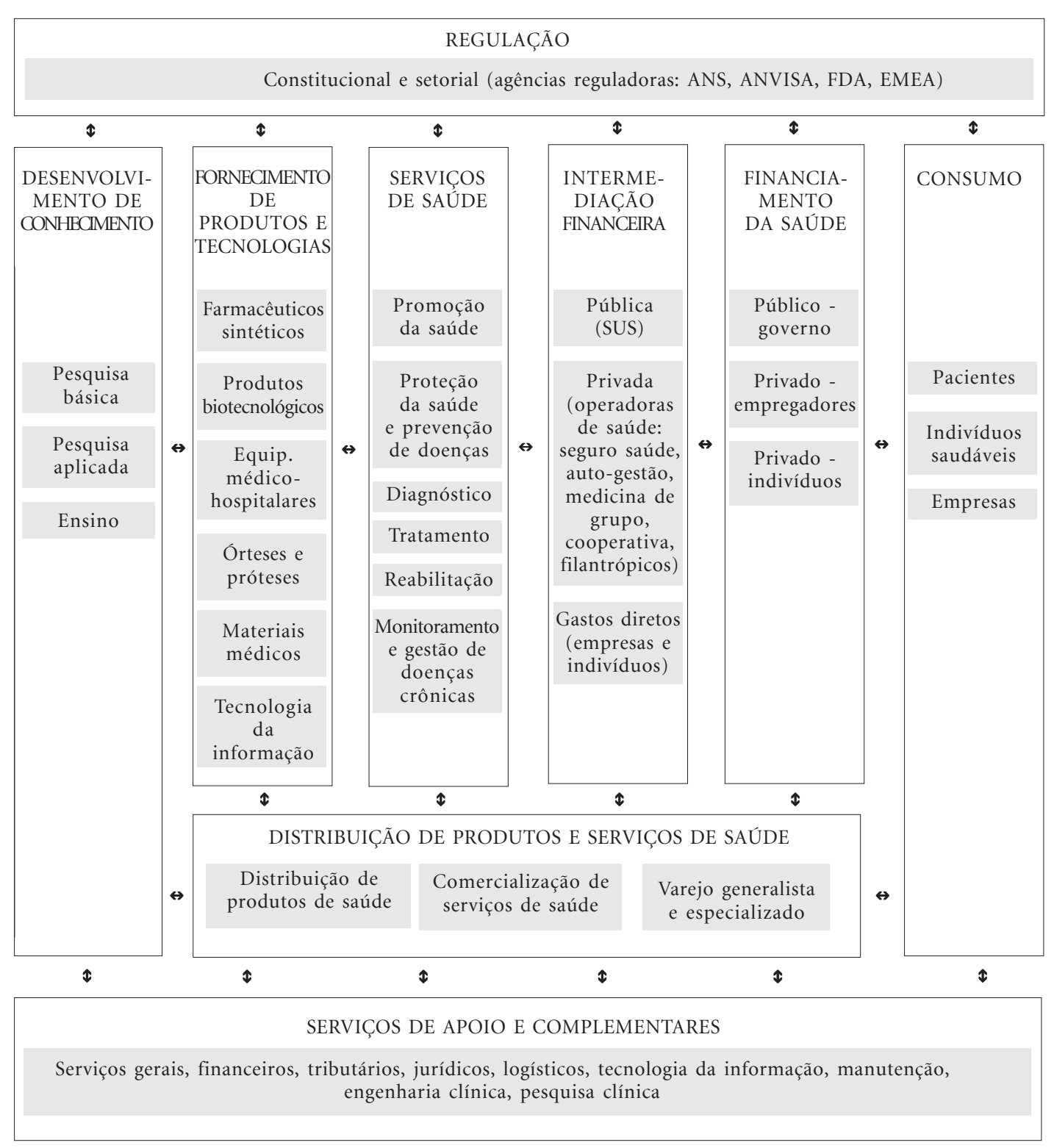

Figura 2. Atividades nos elos da cadeia de valor da saúde (Pedroso ${ }^{19}$ ).

Cadeia de valor da saúde: principais fluxos

Os fluxos da cadeia de valor representam elementos de entrada e saída em cada um dos seus elos (e, dentro destes, das atividades envolvidas), que se propagam em determinados sentidos de modo a atingir determinados objetivos. No caso da cadeia de valor da saúde, os fluxos contemplam quatro elementos principais - inovação e conhecimento; capital; informações; produtos e serviços - que, na forma de entradas e saídas, fluem de maneira bidirecional nos nove elos (Figura 1). Esses fluxos se propagam da porção inicial da cadeia até a porção final, e vice-versa. Cada um deles é descrito a seguir.

\section{Fluxo de inovação e conhecimento}

O fluxo de inovação e conhecimento representa a propagação das inovações e dos conhecimentos de saúde entre os elos da cadeia de valor. A principal direção de propagação ocorre da 
porção inicial para a final da cadeia ${ }^{11}$. Exemplos desse fluxo podem ser encontrados na indústria farmacêutica, em que as empresas atuam como disseminadoras de conhecimento a médicos e pacientes $^{29}$.

O fluxo de inovação e conhecimento é crítico para o desempenho da cadeia de valor da saúde. A inovação tecnológica no setor é considerada um dos principais fatores responsáveis pelo incremento contínuo dos custos da saúde ${ }^{30,31}$. Os investimentos públicos e privados em pesquisa e desenvolvimento na área de saúde são geralmente elevados, quando comparados com outros setores da economia. Por exemplo, nos EUA (em 2003), os principais setores que receberam investimentos públicos em pesquisa e desenvolvimento foram: defesa (em primeiro lugar) e saúde (em segundo $)^{32}$. No setor privado, as empresas farmacêuticas apresentam o maior volume total de investimentos em pesquisa e desenvolvimento, quando comparadas com outros setores ${ }^{13}$.

Não obstante os altos investimentos em pesquisa e desenvolvimento na saúde, a inovação nesse setor geralmente se propaga de maneira lenta e deficiente ${ }^{33}$. O processo de inovação na saúde é complexo em função de diferentes fatores tais como: (1) os diferentes objetivos (por vezes conflitantes) dos atores (ou participantes) da cadeia de valor; (2) as particularidades do financiamento na saúde (público e privado); (3) a regulamentação do setor; (4) a taxa de incorporação de tecnologias em saúde; (5) o crescente aumento do poder dos pacientes nas decisões sobre saúde; e, (6) as constantes pressões para a redução de custos ${ }^{32}$. Além disso, uma regulamentação muito rígida na saúde pode inibir a introdução de inovações de natureza radical no setor $^{34}$. Ressalta-se ainda que grande parte do fluxo de inovações e conhecimentos lida com elementos intangíveis, como por exemplo, o componente tácito do conhecimento (ou seja, aquele conhecimento subentendido e ainda não sistematizado). Apesar do caráter intangível, esse fluxo pode ser analisado e gerenciado de modo a gerar valor para os participantes da cadeia.

\section{Fluxo de produtos e serviços}

O fluxo de produtos e serviços contempla o conjunto de processos e respectivas atividades que unem as entradas e saídas de cada um dos elos (e dentro destes, dos participantes envolvidos) até entregar saúde e bem-estar aos pacientes, aos indivíduos saudáveis e à comunidade em geral. Assim, sua principal direção de propaga- ção ocorre da parte inicial para a parte final da cadeia. O conhecimento tácito representa a principal entrada do primeiro elo, que transforma esses processos em conhecimento codificado (ou explícito). Esse conhecimento é utilizado no elo seguinte, que o incorpora em produtos e tecnologias, utilizados e alocados nos serviços de saúde no terceiro elo. Este entrega os serviços aos pacientes e indivíduos saudáveis (sexto elo) e é remunerado por meio do quarto elo (intermediação financeira) que, por sua vez, recebe os recursos financeiros provenientes do financiamento da saúde (quinto elo). Os processos e as atividades do fluxo de produtos e serviços são regulados pelo primeiro elo horizontal. A distribuição de produtos e serviços de saúde (segundo elo horizontal) interage com os demais elos verticais e horizontais para realizar os processos logísticos de suprimentos e distribuição, bem como a comercialização dos produtos e serviços. Por fim, os serviços de apoio e complementares (terceiro elo horizontal) interagem com os demais elos verticais e horizontais, com o intuito de apoiar e complementar os processos e as atividades inerentes a cada um deles.

No fluxo de produtos e serviços, é importante analisar como as operações são realizadas. Em particular, no elo dos serviços de saúde, pode-se utilizar a segmentação conforme o nível de complexidade. A esfera de atendimento primário foca nos atendimentos de menor complexidade. Geralmente esse atendimento ocorre nas organizações voltadas à atenção primária (ex.: centros de saúde e ambulatórios gerais), que deveriam funcionar como porta de entrada aos níveis de maior complexidade do sistema. A esfera de atendimento secundário enfatiza o de níveis intermediários de complexidade, como ocorre em ambulatórios com especialidades clínicas e cirúrgicas, serviços de apoio ao diagnóstico e terapia, determinados serviços de atendimento de urgência e emergência e hospitais gerais. A esfera de atendimento terciário foca os de maior complexidade, tais como serviços realizados por hospitais especializados, que atuam no nível regional, estadual ou mesmo nacional ${ }^{35}$.

A Figura 3 ilustra o fluxo de produtos e serviços na cadeia de valor da saúde. Os fluxos de produtos se propagam principalmente na direção do fornecimento de produtos e tecnologias para os consumidores. Os fluxos reversos de produtos (ou seja, os fluxos de produtos no sentido inverso ao principal, tais como devoluções, retornos de itens obsoletos, reparos e reciclagem) são representados pelas setas bidirecionais na figura. 


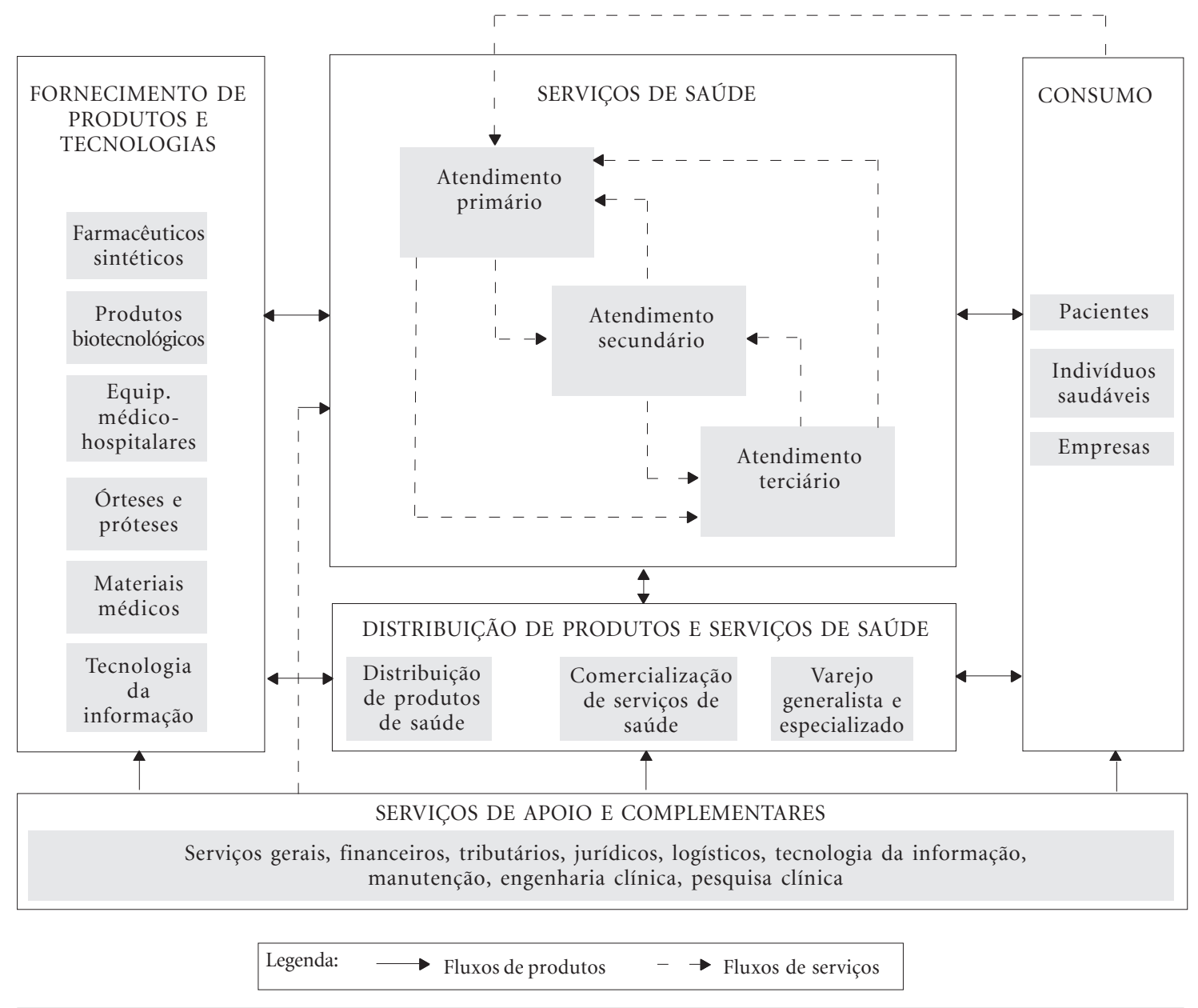

Figura 3. Fluxos de produtos e serviços na cadeia de valor da saúde (Pedroso ${ }^{19}$ ).

Os fluxos principais de serviços de saúde convergem para o elo dos serviços de saúde. Esses fluxos apresentam dois processos polares: (1) processos iterativos de assistência à saúde, em que as atividades são mais customizadas; e (2) processos sequenciais de assistência à saúde, em que as atividades podem ser padronizadas ${ }^{36,37}$. De maneira geral, os processos iterativos são mais adequados ao diagnóstico e tratamento de casos de maior complexidade, enquanto os processos sequenciais podem ser utilizados para a resolução de casos de menor complexidade ${ }^{37}$ e para a assistência coletiva.

Nesse contexto, a esfera de atendimento primário, posicionada como porta de entrada dos serviços de saúde, tende a apresentar processos mais padronizados de cuidados de saúde, para atendimentos de menor complexidade. A de atendimento terciário tende a atuar por meio de proces- sos mais customizados e iterativos, para atendimentos de maior complexidade. A de atendimento secundário geralmente contempla uma composição de processos padronizados e customizados, para atendimentos de complexidade intermediária. Os consumidores percorrem as esferas de atendimento conforme suas necessidades de serviços de saúde e complexidade do atendimento.

\section{Fluxo financeiro}

O fluxo financeiro representa a propagação - e a retenção - do capital ao longo da cadeia de valor. Ao contrário dos anteriores, sua direção principal de propagação ocorre da parte final ou, mais especificamente, do financiamento para a porção inicial da cadeia.

A Figura 4 apresenta os fluxos financeiros na cadeia de valor da saúde. Nessa figura, o merca- 




Figura 4. Fluxos financeiros na cadeia de valor da saúde (Pedroso $\left.{ }^{19}\right)$.

do é considerado como um conjunto de transações financeiras. Nesse contexto, há quatro mercados na cadeia de valor da saúde: (1) mercado financeiro da saúde (cujos recursos são alocados diretamente pelo governo, empregadores e indivíduos, e indiretamente por meio do pagamento de impostos); (2) mercado de oferta de serviços, produtos e tecnologias de saúde; (3) mercado de consumo de saúde; e, (4) mercado de conhecimento em saúde (onde o conhecimento é remunerado, tal como ocorre no pagamento de royalties e em determinadas atividades de educação).

Esses quatro mercados são regulados por agentes, por meio de quatro conjuntos principais de políticas e regras: (1) financiamento da saúde (ex.: políticas de financiamento do sistema público); (2) alocação dos recursos de saúde (ex.: políticas de distribuição das verbas de saúde); (3) utilização dos recursos de saúde (ex.: políticas de vacinação do sistema público, regras de utilização do sistema de saúde suplementar); (4) monetização (ou mercantilização) do conhecimento em saúde (ex.: regras de patentes e propriedade intelectual).

A análise do fluxo financeiro pode ser utilizada para mapear ineficiências na cadeia de valor, verificar quais elos apresentam maior valor financeiro adicionado ou identificar padrões de consumo de saúde. Na primeira questão, é importante notar que ineficiências em elos anteriores podem se propagar aos posteriores, resultando em ineficiências sistêmicas. Assim, aumentos nos custos médico-hospitalares percebidos na intermediação financeira podem ser causados por ineficiências localizadas nos elos anteriores (por exemplo, nos serviços de saúde e no fornecimento de produtos e tecnologias), quando as empresas incorporam suas ineficiências aos preços dos produtos e serviços (por exemplo, como uma justificativa ao aumento de seus custos).

Nos EUA, há importantes variações regionais nos custos de saúde, não explicadas somente por 
diferenças na disponibilidade de recursos tecnológicos e na forma de pagamentos. Nesse sentido, alguns autores sugerem que a reorganização dos serviços de saúde e a eliminação de serviços desnecessários poderiam melhorar a eficiência do sistema e, portanto, reduzir o crescimento dos $\operatorname{custos}^{38}$. Vale ressaltar que os custos da saúde podem causar importantes impactos no elo do financiamento da saúde.

No que diz respeito à agregação de valor na cadeia, Campos et al. ${ }^{17}$ verificaram que médicos, indústria farmacêutica, hospitais privados e empresas de medicina diagnóstica, nessa ordem, são os atores que geram o maior valor financeiro adicionado na cadeia de saúde do Brasil. Em relação aos padrões de consumo de saúde, sabe-se que a adoção de políticas que aumentam o compartilhamento de custos com os consumidores altera suas decisões quanto às opções por provedores de serviços de saúde, aderência e escolha de custos de tratamento. Verifica-se que essas políticas (por exemplo, coparticipação e franquia) reduzem o consumo e os custos de saúde. Por outro lado, os consumidores tendem a economizar também na utilização de produtos e serviços essenciais de saúde, quando o compartilhamento de custos é excessivo ${ }^{39,40}$.

\section{Fluxo de informação}

O fluxo de informação representa a propagação de informações entre as atividades da cadeia de valor da saúde. Cinco fluxos principais podem ser identificados: (1) oferta e produção de produtos, serviços e tecnologias; (2) demanda de produtos, serviços e tecnologias; (3) disponibilidade financeira e acesso; (4) aspectos regulatórios; (5) conhecimento em saúde. Nesse contexto, os sistemas de informação em saúde podem ser utilizados para coordenar os fluxos de informação, bem como armazenar, organizar e disponibilizar os dados para as instituições, as empresas e os indivíduos que participam do sistema de saúde (Figura 5).

A informação sobre oferta e produção de produtos, serviços e tecnologias aborda dados sobre: (1) oferta em saúde, que inclui informações sobre os prestadores de serviços, fornecedores e distribuidores de produtos e tecnologias (ex.: localização, especialização, capacidade, preços); (2) produção em saúde, que contempla as informações sobre produção de produtos e serviços, bem como o desempenho de prestadores e fornecedores. Segundo proposta de alguns pesquisadores $^{12,21,41}$, a visibilidade dessas informa- ções (ex.: divulgação de preços e resultados) poderia levar a um aumento da competição no setor e, consequentemente, gerar um estímulo à melhoria de qualidade e eficiência.

O fluxo de informação sobre demanda contempla as necessidades (inclusive aquelas não atendidas) e o consumo de serviços, produtos e tecnologias por parte dos integrantes do sistema de saúde. Nesse fluxo, a propagação das necessidades e do consumo dos usuários aos elos anteriores da cadeia pode permitir maior visibilidade da demanda real do sistema de saúde. Assim, dados epidemiológicos poderiam ser obtidos a partir das informações sobre o consumo de produtos e serviços. No sentido inverso, também poderiam ser utilizados para estimar a demanda de produtos e serviços (adicionalmente à avaliação dos riscos ou dos indicadores de serviços já em uso). Por exemplo, a informação sobre a população de doentes renais crônicos em determinada região pode ser utilizada para estimar a demanda potencial de diferentes tipos de terapia renal substitutiva (ex.: hemodiálise, diálise peritoneal e transplante renal).

O fluxo de informação sobre disponibilidade financeira e acesso considera dois tipos de informação. A primeira consiste nas informações sobre a disponibilidade e a alocação de recursos financeiros às atividades de prestação de serviços, fornecimento e distribuição de produtos e tecnologias e desenvolvimento de conhecimento em saúde. A segunda considera as regras de utilização e o acesso dos consumidores, bem como as atividades de autorização das operadoras de planos de saúde (no papel de participantes da intermediação financeira) aos prestadores de serviços, e fornecedores e distribuidores de produtos e tecnologias.

O fluxo de informação sobre os aspectos regulatórios contempla a disponibilização e o acesso às regulamentações inerentes à produção de produtos e serviços de saúde. Também se volta às informações referentes às ações de fiscalização, controle, avaliação e auditoria, que deveriam assegurar o cumprimento dessas regulamentações ${ }^{42}$.

O fluxo de conhecimento aborda a geração, a organização, a disponibilização e o acesso ao conhecimento em saúde. Esse fluxo é relevante no setor, pois a saúde é uma área intensiva em conhecimento quando comparada com outros setores da economia, estando o conhecimento em plena evolução. Por exemplo, cerca de 30 mil novas citações são inseridas a cada mês no Medli$n \mathrm{e}^{37}$, banco de dados on line sobre periódicos médicos. 
OFERTA E PRODUÇÃO (PRODUTOS, SERVIÇOS E TECNOLOGIAS)



Figura 5. Fluxos de informação na cadeia de valor da saúde (adaptado de Pedroso ${ }^{19}$ ).

No contexto dos sistemas de informação em saúde, ressalta-se o prontuário eletrônico do paciente, um sistema com informações integradas sobre sua saúde. Esse sistema armazena e gerencia as informações de saúde dos indivíduos, bem como provê acesso e intercâmbio dessas informações por diversos integrantes do sistema de saúde ${ }^{43}$.

\section{Discussão}

A cadeia de valor da saúde apresentada neste trabalho teve como propósito uma tentativa de aproximação do modelo teórico à realidade brasileira. Além disso, a caracterização da cadeia por meio de atividades permite maior generalização e flexibilidade de utilização.

Os autores entendem que os noves elos adotados - seis verticais e três horizontais - representam, de maneira abrangente, a cadeia de valor do setor de saúde do Brasil. Os quatro fluxos principais (inovação e conhecimento, produtos e serviços, financeiro e informação) abordam diferentes variáveis, geralmente interdependentes, de gestão. A consistência na integração e a coerência no alinhamento entre os quatro fluxos e suas variáveis podem contribuir para incrementar a geração de valor econômico e social entregue aos consumidores e à sociedade.

A cadeia de valor da saúde é fragmentada, tanto nos Estados Unidos ${ }^{12,22}$ como no Brasil ${ }^{19}$, o que aumenta a complexidade de sua gestão. Assim, a análise desses fluxos pode fornecer importantes elementos para a compreensão dos relacionamentos, a verificação do alinhamento de incentivos, bem como a identificação de ineficiências nas interfaces entre elos ou entre organizações. Na saúde suplementar, por exemplo, um ponto típico de conflito ocorre na interface entre as operadoras e os provedores de serviços de saúde. Em grande parte, esse fato acarreta ineficiências sistêmicas quando cada uma das partes (operadoras e provedores) atua para atender 
seus objetivos, em detrimento da melhoria do sistema como um todo.

No SUS, um relatório do Banco Mundial apontou inadequação da estrutura de incentivos. Segundo esse estudo, o SUS opera com muitos incentivos embutidos e implícitos - inerentes à complexidade do sistema -, que geram ineficiências ou má qualidade da assistência à saúde ${ }^{44}$. Certamente houve destacados avanços nesse sistema desde que foi instituído, em 1988. Não obstante, ainda há importantes desafios pela frente, particularmente ao compararmos com outros sistemas universais consolidados há mais tempo, tais como aqueles encontrados na Alemanha, Canadá, Japão e Reino Unido ${ }^{24}$. Nesse contexto, a análise dos quatro fluxos entre as atividades da cadeia de valor e os “atores” por elas responsáveis pode contribuir para um melhor alinhamento nos incentivos das organizações que atuam no SUS e, consequentemente, uma melhoria no desempenho do sistema como um todo.

Consideramos que o modelo proposto neste trabalho pode ser adotado em atividades acadêmicas (ex.: ensino e pesquisa) e estudos do setor de saúde do Brasil. Em particular, a cadeia de valor da saúde pode ser utilizada para a análise e a proposição de soluções para problemas de natureza sistêmica, tais como aqueles relacionados à melhoria de coordenação entre atividades de assistência à saúde, da estrutura de incentivos e do relacionamento entre participantes do sistema de saúde.

\section{Colaboradores}

MC Pedroso trabalhou na concepção do modelo, revisão da literatura e redação final; AM Malik atuou na revisão crítica. Ambos os autores foram responsáveis pela aprovação da versão a ser publicada. 


\section{Referências}

1. Porter MA. What is value in health care? $N$ Engl J Med 2010; 363(26):2477-2481.

2. Peteraf MA, Barney JB. Unraveling the resource-based triangle. Manag Dec Econ 2003; 24(4):309-323.

3. World Health Organization (WHO). The world health report 2000: health systems: improving performance. Geneva: WHO; 2000.

4. Brasil. Constituição da República Federativa do Brasil. Brasília: Senado Federal; 1988.

5. Porter ME, Kramer MR. Creating shared value. Harvard Bus Rev 2011; 89(1/2):62-77.

6. Porter ME. Competitive advantage: creating and sustaining superior performance. New York: Free Press; 1985.

7. Pedroso MC. Um estudo sobre o desenvolvimento das competências em gestão da cadeia de suprimentos [tese]. São Paulo: Universidade de São Paulo; 2002.

8. Almeida Filho N, Jucá V. Saúde como ausência de doença: crítica à teoria funcionalista de Christopher Boorse. Cien Saude Colet 2002; 7(4):879-889.

9. World Health Organization (WHO). Constitution of the World Health Organization. Basic documents. Forty-fifth edition, supplement. Geneva: WHO; 2006.

10. Burns LR, Degraaf RA, Danzon PM, Kimberly JR, Kissik WL, Pauly MV. The Wharton School study of the health care value chain. In: Burns LR, editor. The health care value chain: producers, purchasers and providers. San Francisco: Jossey-Bass; 2002. p. 3-26.

11. Burns LR. The business of healthcare innovation in the Wharton School curriculum. In: Burns LR, editor. The business of health care innovation. New York: Cambridge University Press; 2005. p. 1-23.

12. Porter ME, Teisberg EO. Redefining health care: creating value-based competition on results. Boston: Harvard Business School Press; 2006.

13. Christensen CM, Grossman JH, Hwang J. The innovator's prescription: a disruptive solution for health care. New York: McGraw-Hill; 2009.

14. Pitta DA, Laric MV. Value chains in health care. $J$ Cons Mark 2004; 21(7):451-464.

15. Pisano GP. Science business: the promise, the reality and the future of biotech. Boston: Harvard Business School Press; 2006.

16. Gadelha CAG. O complexo industrial da saúde e a necessidade de um enfoque dinâmico na economia da saúde. Cien Saude Colet 2003; 8(2):521-535.

17. Campos EF, Gontijo MCF, Oliveira PJ, Chaves SR, Laudares P, editores. A cadeia de valor em saúde: uma proposta de reorganização da atenção na saúde suplementar. Belo Horizonte: Unimed Federação Minas; 2009.

18. Walters D, Jones P. Value and value chains in healthcare: a quality management perspective. The TQM Mag 2001; 13(5):319-333.

19. Pedroso MC. Um modelo de gestão estratégica para serviços de saúde [tese]. São Paulo: Universidade de São Paulo; 2010.

20. Herzlinger RE, editor. Consumer-driven health care: implications for providers, payers, and policy-makers. Boston: Harvard Business School Publishing; 2004.
21. Herzlinger RE. Who killed health care? America's \$2 trillion medical problem - and the consumer-driven cure. New York: McGraw-Hill; 2007.

22. Shi L, Singh DA. Delivering health care in America: a systems approach. 4th. Ed. Sudbury: Jones and Bartlett Publishers; 2008.

23. World Health Organization (WHO). World health statistics 2010. Geneva: WHO; 2010.

24. Bodenheimer TS, Grumbach K. Understanding health policy: a clinical approach. 5th. Ed. New York: McGraw-Hill; 2005.

25. Santos IS, Ugá MAD, Porto SM. O mix públicoprivado no Sistema de Saúde Brasileiro: financiamento, oferta e utilização de serviços de saúde. Cien Saude Colet 2008; 13(5):1431-1440.

26. Bloche MG. Health care for all? N Engl J Med 2007; 357(12):1173-1175.

27. Anderson GF, Reinhardt UE, Hussey PS, Petrosyan V. It's the prices, stupid: why the United States is so different from other countries. Health Aff 2003; 22(3):89-105.

28. Pacheco RS. Regulação no Brasil: desenho das agências e formas de controle. Rev Adm Pública 2006; 40(4):523-543.

29. Collier J, Iheanacho I. The pharmaceutical industry as an informant. Lancet 2002; 360(9343):1405-1409.

30. Cutler DM, McClellan M. Is technological change in medicine worth it? Health Aff 2001; 20(5):11-29.

31. Bodenheimer T. High and rising health care costs. Part 2: technological innovation. Ann Intern Med 2005; 142(11):932-937.

32. Herzlinger RE. Why innovation in health care is so hard. Harvard Bus Rev 2006; 84(5):58-66.

33. Berwick DM. Disseminating innovations in health care. JAMA 2003; 289(15):1969-1975.

34. Curtis LH, Schulman KA. Overregulation of health care: musings on disruptive innovation theory. Law Contemp Probl 2006; 69(4):195-206.

35. Cecílio LCO. Modelos tecno-assistenciais em saúde: da pirâmide ao círculo, uma possibilidade a ser explorada. Cad Saude Publica 1997; 13(3):469-478.

36. Bohmer RMJ. Medicine's service challenge: blending custom and standard care. Health Care Manag Rev 2005; 30(4):322-330.

37. Bohmer RMJ. Designing care: aligning the nature and management of health care. Boston: Harvard Business Press; 2009.

38. Fisher ES, Bynum JP, Skinner JS. Slowing the growth of health care costs - lessons from regional variation. N Engl J Med 2009; 360(2):849-852.

39. Buntin MB, Damberg C, Haviland A, Kapur K, Lurie N, McDevitt R, Marquis MS. Consumer-directed health care: early evidence about effects on cost and quality. Health Aff 2006; 25(6):516-530.

40. Rosenthal MB. What works in market-oriented health policy? N Eng J Med 2009; 360(21):2157-2160.

41. Pedroso MC, Malik AM. As quatro dimensões competitivas da saúde. Harvard Bus Rev. Ed. Brasil. 2011; 89(3):54-63.

42. Mendonça CS, Reis AF, Moraes JC, organizadores. A política de regulação do Brasil. Brasília: Organização Pan-Americana da Saúde; 2006. 
43. Blumenthal D, Glaser JP. Information technology comes to medicine. N Engl J Med 2007; 356(24):25272534.

44. World Bank. Governance in Brazil's Unified Health System (SUS). Raising the quality of public spending and resource management. Report No. 36601-BR. February 15, 2007 [site na Internet]. [acessado 2011 mar 22]. Disponível em: http://www.bancomundial. org.br/index.php/content/view_folder/88.html.

Artigo apresentado em 10/03/2011

Versão final apresentada em 16/07/2011 